\title{
A nonlinear dynamic model of magnetorheological elastomers in magnetic fields based on fractional viscoelasticity
}

\author{
Guanghong Zhu ${ }^{\mathrm{a}^{*}}$, Yeping Xiong ${ }^{\mathrm{b}}$, Zigang $\mathrm{Li}^{\mathrm{a}}$, Ling Xiao ${ }^{\mathrm{a}}$, Ming Li ${ }^{\mathrm{a}}$, Xianxu 'Frank' Bai ${ }^{\mathrm{c}}$ \\ Department of Mechanics, Xi' an University of Science and Technology, Xi'an 710054, China \\ ${ }^{\mathrm{b}}$ Faculty of Engineering and the Physical Sciences, University of Southampton, Southampton SO16 \\ 7QF, UK \\ ${ }^{c}$ Laboratory for Adaptive Structures and Intelligent Systems (LASIS), Department of Vehicle \\ Engineering, Hefei University of Technology, Hefei 230009, China \\ *Corresponding authors. \\ E-mail addresses: zhuguanghong@ $@$ xust.edu.cn (G. Zhu)
}

\begin{abstract}
As smart materials, magnetorheological elastomers (MREs) have been broadly applied in the field of intelligent structures and devices. In order to mathematically represent the dynamic behavior in a wide range of strain amplitude, excitation frequency and magnetic field; a nonlinear model with a fractional element was developed for MREs in a linear viscoelastic regime. The identification of model parameters was realized through fitting experimental data of dynamic moduli measured in shear mode, and the identified parameters exhibited good repeatability and consistency to reflect the rationality of this nonlinear dynamic model. Considering material elasticity and viscosity, the dependence of model parameters on strain amplitudes and magnetic fields was analysed to interpret the dynamics of MREs. The fitted results displayed an excellent agreement with the experimental results on the dependence of dynamic moduli on strain amplitudes and magnetic fields. Using the predictor-corrector approach, predicted results on the stress-strain hysteresis loop were calculated based on identified parameters to further validate the proposed model by comparing with experimental results and predicted results of the revised Bouc-Wen model. This proposed model is expected to facilitate the dynamic analysis and simulation of MRE based vibration systems with a high precision accuracy.
\end{abstract}

Keywords: nonlinear dynamic model, magnetorheological elastomers, fractional viscoelasticity, dynamic mechanical analysis, stress-strain hysteresis

\section{Introduction}

Magnetorheological elastomers (MREs) consist of magnetizable particles suspended in an elastomer matrix, and anisotropic MREs have a definite orientation of particles due to the solidification in the presence of magnetic fields (Kaleta et al., 
2011; Sapouna et al., 2017). Over the past decades MREs have attracted increasing attention to the reduction of undesired vibrations. Increasing efforts have been devoted to the application of smart materials for semi-active/adaptive-passive vibration control which owns both the reliability of passive control and the versatility of active control (Daley and Wang, 2008; Fu et al., 2017). Because of the controllable rheological property, MRE is a promising smart material in practice (Ibrahim 2008; Xing et al., 2015; Yuan et al., 2019) and has been applied broadly in controllable absorbers and isolators (Leng et al., 2019; Lerner and Cunefare, 2007; Nguyen et al., 2018; Sun et al., 2017; Xin et al., 2017).

The dynamic behavior of MREs can be affected by not only the material themselves, including components and fabrications, but also by in-service conditions (Bai et al., 2019; Wan et al., 2019; Wang and Kari, 2019;); so the development of the dynamic model for MREs is a foremost problem for the realization of vibration control. Since the first dipole model of MREs, chain and column models have been subsequently proposed for MREs, to optimize the particle volume fraction and predict the field-induced modulus (Chen et al., 2007; Davis, 1999; Jolly et al., 1996). Considering these models are basically quasi-static, Blom and Kari (2011) developed a constitutive model in an audible frequency range integrating the amplitude dependence, which is commonly disregarded for static, quasi-static and dynamic models with small amplitudes. Their research accepted microscopic dipolar approximation approach and failed to depict the dynamic behavior of MREs comprehensively in magnetic fields (Graczykowski and Pawlowski, 2017).

As classical models of viscoelastic materials, the Kelvin-Voigt model and the Maxwell model can describe the creep and the relaxation phenomena, respectively; and the Zener model comprises of a spring in parallel with a Maxwell model. These simple models fail to represent the viscoelastic behavior of MREs with respect to broad frequency ranges. Based on classical viscoelastic models, generalized Maxwell models can represent the dynamic behavior more effectively by increasing the number of components, but as a result the parameter evaluation becomes difficult. As MREs exhibit hysteresis, Bouc-Wen model (Behrooz et al., 2014; Yang et al., 2013), Ramberg-Osgood model (Eem et al., 2012) and Prandtl-Ishlinskii model (Dargahi et al., 2019) were developed to represent the hysteretic properties, and the parameters were iteratively calculated with trust region reflective algorithms or evolutionary algorithms. Most of classical material models cannot accurately depict the dynamic 
behavior of MREs across a wide range of excitation frequency and strain amplitudes, and the fractional derivative has been introduced to express the stress-strain relation of viscoelastic materials (Nguryen et al., 2020; Sasso et al., 2011). The material stiffness becomes high at high frequencies or small amplitudes, and decreases with reducing frequencies or increasing excitation amplitudes (Kumbhar et al., 2018; Yu et al., 2015). Recently, the efficiencies were compared between several fractional rheological models for MREs, and the description of dynamic behavior for a wide range of magnetic fields can be realized with two fractional elements, which can be interpreted as the contributions of magnetizable particles and polymer network to material elasticity and viscosity (Nadzharyan et al., 2018; Poojary and Gangadharan, 2018). The researchers have achieved an early development of dynamic models for MREs, however, as an essential step towards the realization of its potential for vibration control, effective dynamic models with a great accuracy deserve more effort.

In this study, the experimental results of dynamic moduli are acquired by performing dynamic mechanical analysis (DMA) tests in shear mode and a nonlinear dynamic model is developed by further rationalizing a fractional-order model (Zhu et al., 2020). Using the genetic algorithm (GA) to optimize errors, the model parameters are identified by fitting the experimental data on frequency dependence of dynamic moduli, and the identified parameters exhibit a very small deviation. Being different from the previous work (Zhu et al., 2020), this nonlinear dynamic model is proved to be highly reliable with a good consistency and repeatability of identified parameters, which allows a detailed observation of the material elasticity and viscosity. The model parameters are analyzed by varying magnetic fields and strain amplitudes to interpret the dynamics of MREs, and the fitted results are compared with the experimental results on the strain amplitude and magnetic field dependence of dynamic moduli. By using the predictor-corrector approach, the numerical solutions of this nonlinear dynamic model are calculated to predict the stress-strain hysteresis loop. Comparing with the previous work (Zhu et al., 2020), the accuracy of predicted results for hysteresis phenomenon is improved obviously in this proposed nonlinear dynamic model, which indicates a good applicability of this nonlinear dynamic model for MREs. Eventually, The predicted results on the stress-strain hysteresis loop are compared with both the experimental results and predicted results of the revised 
Bouc-Wen model (Wang et al., 2018) to evaluate the efficiency of this proposed nonlinear dynamic model.

\section{Modeling of MREs}

\subsection{Dynamic mechanical analysis}

When the excitation is sinusoidal, the stress response of viscoelastic materials will lag behind the strain input by an angle $\varphi$, which is called loss angle and ranges from 0 to $90^{\circ}$. The contributions of the strain to the in-phase and out-of-phase stress can be expressed by a complex modulus $M^{*}$

$$
M^{*}=M^{\prime}+\mathrm{i} M^{\prime \prime}
$$

where the modulus $M$ can be either the Young's modulus $E$ or the shear modulus $G$. The storage modulus $M^{\prime}$ indicates the ability of storing energy due to deformation, and the loss modulus $M^{\prime \prime}$ indicates the ability of dissipating energy due to deformation.

To describe the efficiency of damping for viscoelastic materials, the loss factor $\tan \varphi$ is introduced as a commonly used term, which is defined to be the ratio of the loss modulus to the storage modulus. The loss angle is relevant to the energy dissipation within an oscillatory cycle, and it can be calculated according to the energy method for loss angle determination in DMA calculation of WaveMatrix software reference manual.

$$
=\arcsin \frac{E_{l p}}{A_{s n} A_{s s}}
$$

where $A_{s n}$ denotes the strain amplitude, $A_{s s}$ is for the stress amplitude, and $E_{l p}$ indicates the energy enclosed by a stress-strain hysteresis loop which can be calculated with numerical integration. When the complex modulus $M^{*}$ is defined to be the ratio between the stress range and the strain range, the storage modulus $M^{\prime}$ and the loss modulus $M^{\prime \prime}$ can be expressed as

$$
\begin{aligned}
& M^{\prime}=M \cos \\
& M^{\prime \prime}=M \sin
\end{aligned}
$$

\subsection{Nonlinear dynamic model}

The constitutive equation of viscoelastic materials can be used to depict the dynamic behavior of MREs under harmonic loads. Simple Voigt models comprising a viscous dashpot and a spring connected in parallel are widely used to represent the 
creep, where the deformation gradually increases along time under unchanged stress; while simple Maxwell models consisting of a viscous dashpot and a spring connected in series are mostly used to represent the relaxation, where the stress gradually decreases along time under unchanged deformation. Fractional elements were introduced to describe the dynamic behavior of MREs, which perform between elastic and viscous behaviors ( $\mathrm{Li}$ and Deng, 2007). The relationship between the shear stress and the shear strain can be expressed as

$$
{ }_{v}=G_{v}{ }_{0} D[(t)] 0<<1
$$

where $G_{v}, \tau_{v}$ and $\gamma_{v}$ are the shear modulus, the shear stress and the shear strain of this fractional model, respectively; $\tau_{0}$ denotes the time constant of relaxation; $D$ is a derivative operator; and $\alpha$ is a fractional-order. As extreme cases, when $\alpha=0$ or $\alpha=1$ the fractional element becomes a spring or a Newton dashpot, respectively.

In the presence of external magnetic fields, a field-induced modulus in MREs will be generated by the resultant interaction between magnetic particles. When the external magnetic field is absent, the field-induced modulus is zero; within a viscoelastic model, a spring connected in parallel can be used to represent the field-induced modulus. Quadratic functions of magnetic flux density are widely used to express the field-induced modulus (Chen et al., 2007; Davis, 1999; Jolly et al., 1996). Although when the restructuring of magnetized particles happens, the best fitting is realized with two-fractional-element models for a broad range of magnetic fields (Nadzharyan et al., 2018); considering the degree of calculations and simulations, the requirement of parameter identifications is also an important factor for the application of dynamic models.

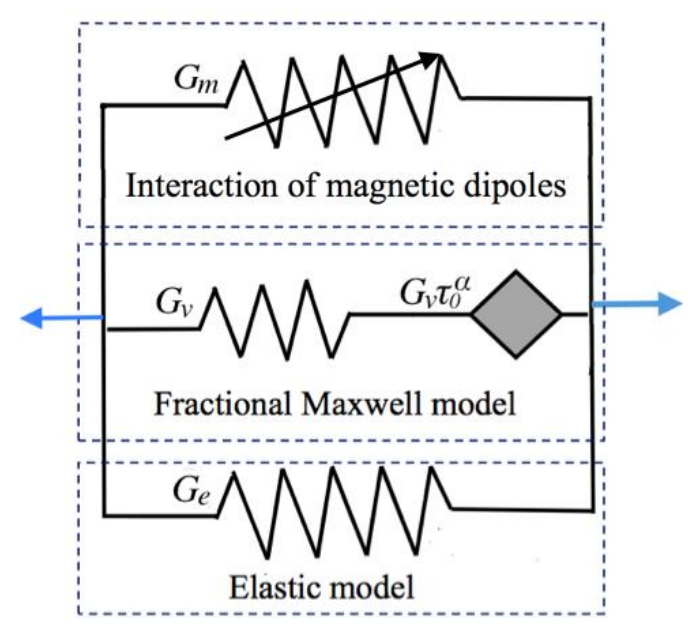

Figure 1 Dynamic model of MRE. 
Aiming at describing the dynamic behavior of MRE with high precision accuracy, the nonlinear dynamic model requiring fewer parameters is developed in Figure 1 by improving a fractional-order model (Zhu et al., 2020), where a elastic model and a fractional Maxwell model are combined to depict the nominal elasticity and viscoelasticity. Integrating the interaction of magnetic particles into the nonlinear dynamic model, another spring component is arranged in parallel to represent the field-induced modulus generated with the application of external magnetic fields. The field-induced shear stress $\tau_{m}$ can be expressed as

$$
{ }_{m}=G_{m}(t)
$$

where $\gamma$ is the shear strain of this nonlinear dynamic model, and the value of $G_{m}$ is 0 in case of no applied magnetic field. Within an idealized range of magnetic field for vibration control, the field-induced shear modulus can be increased effectively as the magnetic flux density increases, and the increase of field-induced shear modulus generally slows down with the magnetic flux density until the magnetic saturation.

In this nonlinear dynamic model, the relationship between the resultant shear stress and the input shear strain can be expressed in the following form:

$$
(t)=G_{e}(t)+G_{m}(t)+\frac{G_{v} D}{1+{ }_{0} D}(t)
$$

When the sinusoidal strain is smaller than $6 \%$ with certain amplitude, the MR effect is assumed to be a mean performance during a vibration period. Considering the MR effect varies with vibration amplitudes when the applied magnetic field is in a steady state, the field-induced modulus can be simplified by a function of strain amplitude and magnetic field. Consequently, the response of MREs to harmonic excitations can be studied with transform methods by converting viscoelastic problems into elastic problems. The Fourier transform is employed herein and the complex shear modulus $G^{*}$ is regarded as a function of frequency. Substituting $\mathrm{i}^{\alpha}=\cos (\alpha \pi / 2)+\mathrm{i} \sin (\alpha \pi / 2)$ into the complex function, the storage modulus (real component) $G^{\prime}$ and the loss modulus (imaginary component) $G^{\prime \prime}$ can be derived in frequency domain:

$$
G^{\prime}()=G_{e}+G_{m}+\frac{G_{v} 0}{\left.1+2 \quad+\cos \frac{}{2}\right)}
$$




$$
G^{\prime \prime}()=\frac{G_{v} \quad 0 \quad \sin \frac{}{2}}{1+2 \sigma_{0} \quad \cos \frac{{ }_{2}}{2}+{ }_{0}^{2}}
$$

In case of the classical Maxwell model $\left(\alpha=1, G_{e}=0\right.$ and $\left.G_{m}=0\right)$ the above quantities can be given as

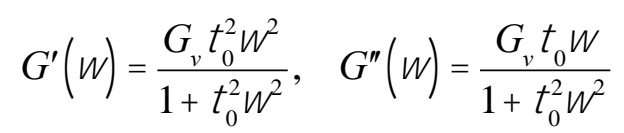

For fractional Maxwell models $\left(G_{e}=0\right.$ and $\left.G_{m}=0\right)$, the shear stress amplitude $\tau_{p}$ and the density of loss energy per cycle $\varepsilon_{v}$ can be determined as

$$
\begin{aligned}
& p=\sqrt{G^{\prime 2}+G^{\prime \prime 2}}=\frac{G_{v 0}}{\sqrt{1+2, \quad \cos \frac{2_{0}}{2}+{ }_{0}^{2}{ }^{2}}} p
\end{aligned}
$$

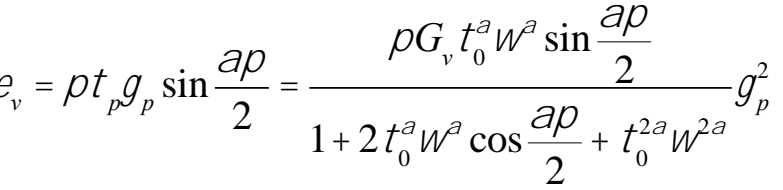

where $\gamma_{p}$ is the shear strain amplitude. When the excitation is harmonic, the stress-strain hysteresis loop response and the loss energy response in steady states are displayed in Figure 2, where the influences of fractional orders on the stress-strain hysteresis loop and the density of loss energy can be observed, respectively.

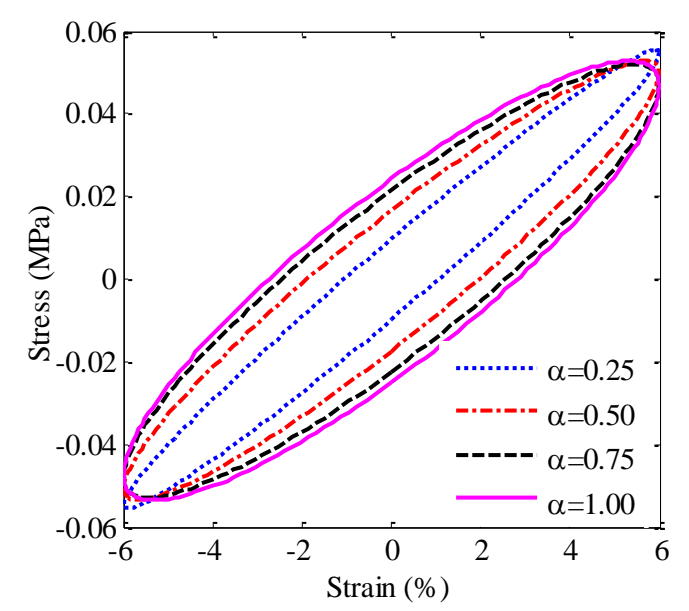

(a)

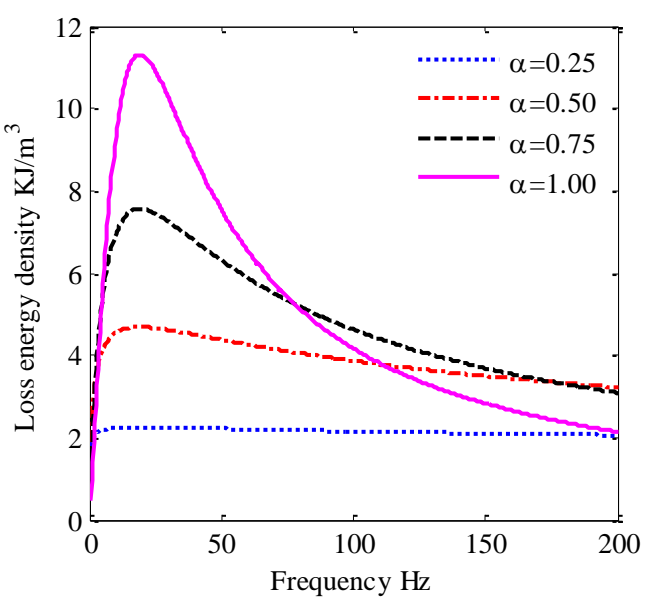

(b)

Figure 2 (a) Viscoelastic stress response and (b) loss energy response at different fractional orders.

\section{Experimental results}

\subsection{Experimental setup}


In this study, the components of anisotropic MRE samples were the silicone rubber (Wacker Chemie AG, Germany) and micron-sized iron powders (Sigma-Aldrich, USA). The solidification underwent with square aluminum molds of dimensions $21.8 \times 21.8 \times 6.5 \mathrm{~mm}^{3}$ and a magnetic field of $290 \mathrm{mT}$ in air dielectric, which was produced by neodymium cylindrical magnets (E-magnets, UK). Taking advantage of the ability of Instron Electropuls E1000 to apply and control the harmonic excitation within a wide frequency range, the DMA tests of MRE samples were conducted in accordance with the BS ISO 4664-1:2011 for shear modes, as shown in Figure 3. The displacement and the force were tracked during tests, and then the dynamic behavior of MRE samples was measured at room temperature (about $25^{\circ} \mathrm{C}$ ) by varying strain amplitudes from $1 \%$ to $6 \%$, frequencies from $1 \mathrm{~Hz}$ to $50 \mathrm{~Hz}$, and magnetic fields from 0 to $500 \mathrm{mT}$.

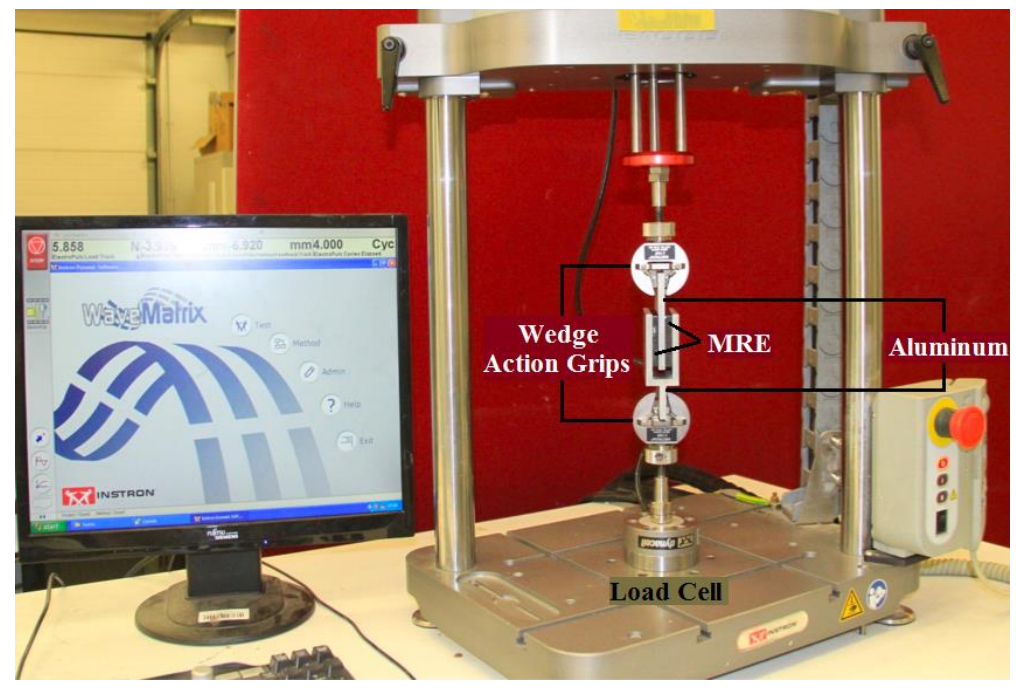

Figure 3 Experimental setup for DMA tests.

\subsection{Dynamic performance}

The experimental data of dynamic moduli is shown in Figure 4, where the storage modulus and loss modulus of MRE seem to decrease with strain amplitudes. The storage modulus increases with the magnetic field, and the increase can be deduced to continue until the magnetic saturation and then the storage modulus remains constant afterwards. The loss modulus also increases with the magnetic field initially, reaches a maximum, and decreases with the magnetic flux density afterwards. The reason is indicated in the Equation (4) where the loss modulus is expressed by the complex modulus and the loss angle; when the magnetic flux density increases, the complex modulus is deemed to increase and the loss angle seems to decrease.

\section{Results and discussion}




\subsection{Parameter identification}

According to Equations (3) and (4), the experimental data of dynamic moduli can be acquired through data processing, while the dynamic moduli can be also fitted according to Equations (8) and (9). The quality of curve fitting can be evaluated with the goodness-of-fit, which is generally measured by calculating the coefficient of determination $R^{2}$ and the standard error ratio $S_{\mathrm{e}} / S_{\mathrm{y}}$ (Yusoff et al., 2011). Using GA for optimization, the model parameters $G_{\mathrm{e}}, G_{\mathrm{v}}, G_{\mathrm{m}}, \tau_{0}$ and $\alpha$ can be identified by minimizing the standard error ratio $S_{\mathrm{e}} / S_{\mathrm{y}}$ of the fitted results on dynamic moduli.

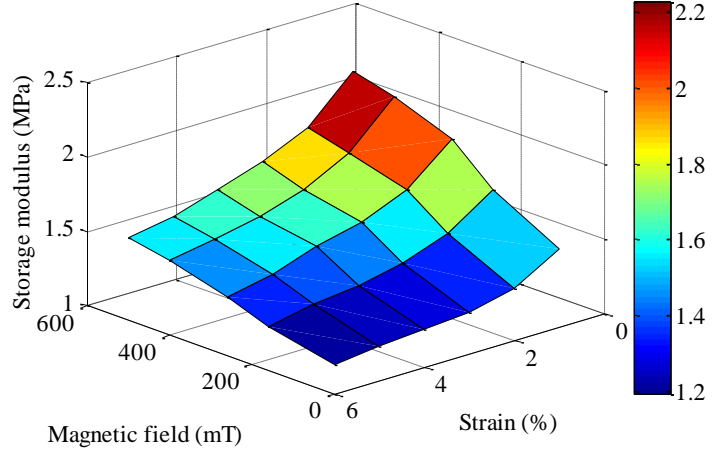

(a)

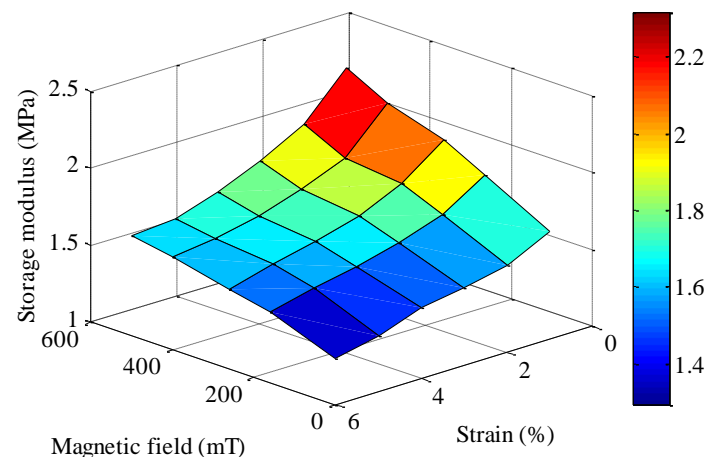

(c)

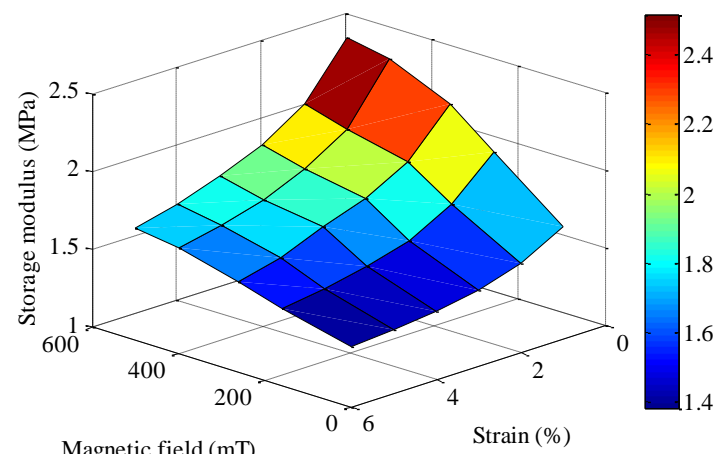

(e)

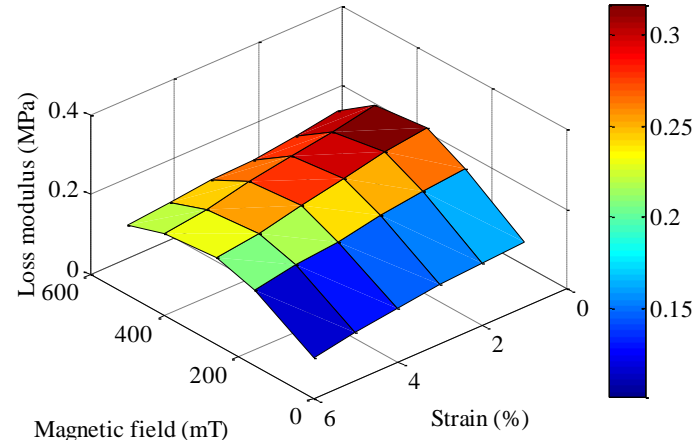

(b)

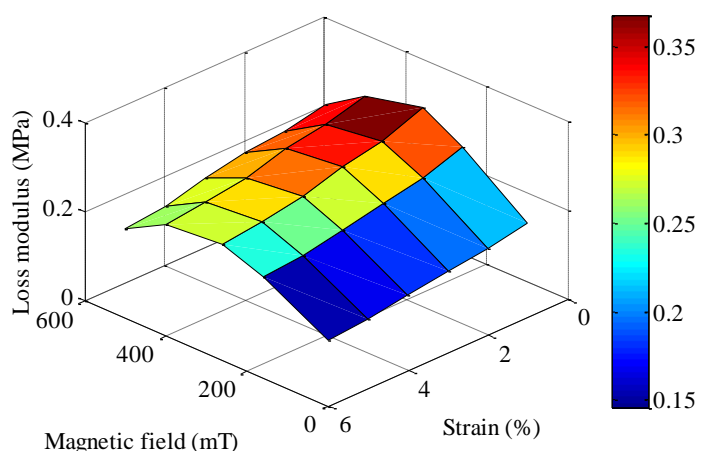

(d)

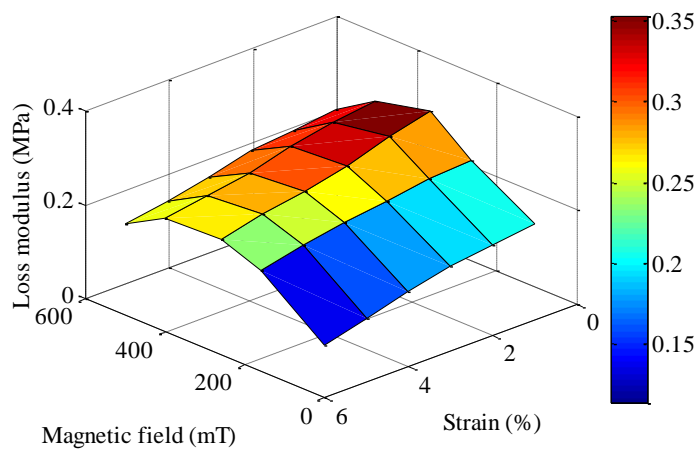

(f)

Figure 4 The magnetic field and strain amplitude dependence of the storage modulus at (a) 1 $\mathrm{Hz}$, (c) $10 \mathrm{~Hz}$, (e) $50 \mathrm{~Hz}$ and the loss modulus at (b) $1 \mathrm{~Hz}$, (d) $10 \mathrm{~Hz}$, (f) $50 \mathrm{~Hz}$. 


$$
\begin{aligned}
& \frac{S_{e}}{S_{y}}=\sqrt{\frac{n\left(y_{\exp } y_{f i t}\right)^{2}}{\left(\begin{array}{ll}
n & 1
\end{array}\right)\left(y_{\exp } Y_{\exp }\right)^{2}}} \\
& R^{2}=1 \frac{n}{n 1 \frac{S_{e}^{2}}{S_{y}^{2}}}
\end{aligned}
$$

where $n$ denotes the number of data samples; $Y_{\exp }$ is the average of experimental results; $y_{\text {exp }}$ and $y_{f i t}$ are the experimental results and fitted results, respectively. The data samples can be either the storage modulus or the loss modulus. When the goodness-of-fit meets the two criteria that the standard error ratio $S_{\mathrm{e}} / S_{\mathrm{y}}<0.35$ and the coefficient of determination $R^{2}>0.90$, the quality of curve fitting can be accepted to be excellent (Yusoff et al., 2011). The details are listed in Table 1.

Table 1 The goodness-of-fit.

\begin{tabular}{llllllll}
\hline \multicolumn{2}{c}{ Strain amplitude $(\%)$} & 1 & \multicolumn{3}{l}{3} \\
Dynamic moduli & $G^{\prime}$ & $G^{\prime \prime}$ & $G^{\prime}$ & $G^{\prime \prime}$ & $G^{\prime}$ & $G^{\prime \prime}$ \\
\hline $0 \mathrm{mT}$ & $R^{2}$ & 0.9985 & 0.9825 & 0.9978 & 0.9968 & 0.9980 & 0.9892 \\
& $S_{\mathrm{e}} / S_{\mathrm{y}}$ & 0.0489 & 0.1791 & 0.0600 & 0.0768 & 0.0583 & 0.1403 \\
$160 \mathrm{mT}$ & $R^{2}$ & 0.9968 & 0.9895 & 0.9988 & 0.9993 & 0.9993 & 0.9772 \\
& $S_{\mathrm{e}} / S_{\mathrm{y}}$ & 0.0728 & 0.1385 & 0.0458 & 0.0346 & 0.0331 & 0.2046 \\
\multirow{2}{*}{$260 \mathrm{mT}$} & $R^{2}$ & 0.9956 & 0.9966 & 0.9996 & 0.9948 & 0.9991 & 0.9954 \\
& $S_{\mathrm{e}} / S_{\mathrm{y}}$ & 0.0854 & 0.0781 & 0.0244 & 0.0979 & 0.0400 & 0.0916 \\
$400 \mathrm{mT}$ & $R^{2}$ & 0.9975 & 0.9615 & 0.9961 & 0.9921 & 0.9988 & 0.9863 \\
& $S_{\mathrm{e}} / S_{\mathrm{y}}$ & 0.0648 & 0.1248 & 0.0806 & 0.1204 & 0.0458 & 0.1584 \\
$500 \mathrm{mT}$ & $R^{2}$ & 0.9963 & 0.9860 & 0.9976 & 0.9998 & 0.9989 & 0.9850 \\
& $S_{\mathrm{e}} / S_{\mathrm{y}}$ & 0.0787 & 0.1603 & 0.0632 & 0.0193 & 0.0435 & 0.1658 \\
\hline
\end{tabular}

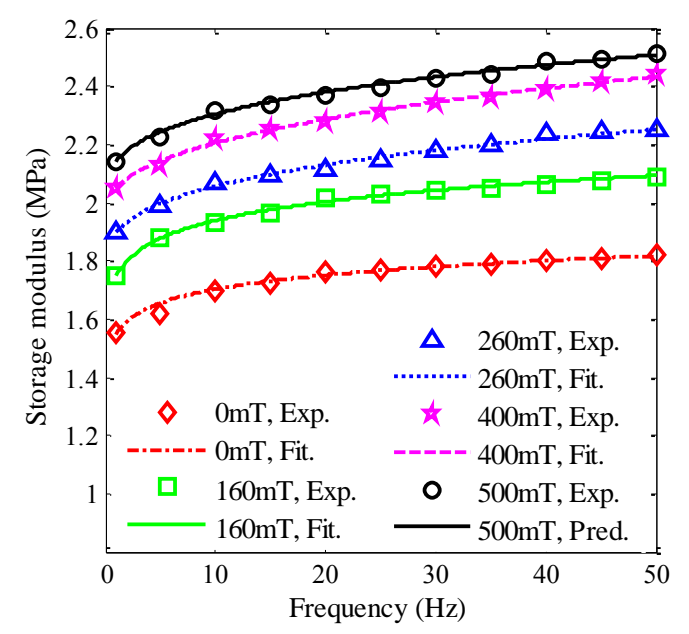

(a)

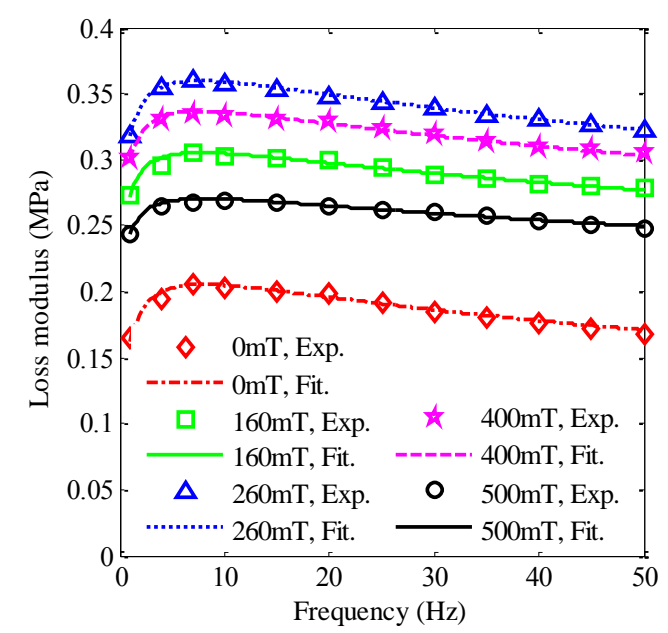

(b)

Figure 5 Comparison between experimental data and fitted results on the frequency dependence of (a) storage modulus and (b) loss modulus with strain amplitude of $1 \%$. 
In steady-state magnetic fields, a set of model parameters $G_{e}, G_{v}, G_{m}, \tau_{0}$ and $\alpha$ can be identified on the frequency dependence of both storage modulus and loss modulus at certain amplitude. These model parameters vary with magnetic flux density and strain amplitude, so the springs in this dynamic model are nonlinear exactly. In Figure 5 , there is an excellent agreement between the experimental results and the fitted results in frequency domain. It can be also observed that the storage modulus increases with the frequency; and the loss modulus increases with the frequency initially, reaches a maximum, and decreases with the frequency afterwards. The frequency dependence of loss modulus is determined by the matrix material, and the shape of curve can be regarded as an inheritance of the matrix material. As for the dependence on magnetic field, the storage modulus of MRE increases with the magnetic flux density, which can be deduced to continue until the magnetic saturation; and the loss modulus increases with the magnetic field initially, reaches a maximum value, and decreases with the magnetic flux density afterwards.

\subsection{Dependency of model parameter}

Comparing with the fractional-order model proposed by Zhu et al. (2020), the parameter identification of this nonlinear dynamic model exhibits better repeatability and consistency. The very small deviation of model parameters allows a further analysis on the elasticity and viscosity of MREs. Figure 6 demonstrates the magnetic field and strain amplitude dependence of the relaxation time constant $\tau_{0}$ and the fractional-order $\alpha$. Both the model parameters $\tau_{0}$ and $\alpha$ increase with the strain amplitude and decrease as the magnetic flux density increases.

The magnetic field and strain amplitude dependence of the elastic shear modulus $G_{\mathrm{e}}$, the viscoelastic shear modulus $G_{\mathrm{v}}$ and the field-induced shear modulus $G_{\mathrm{m}}$ can be observed in Figure 7, where the three model parameters $G_{\mathrm{e}}, G_{\mathrm{v}}$ and $G_{\mathrm{m}}$ decrease as the strain amplitude increases. The elastic shear modulus $G_{\mathrm{e}}$ does not change with magnetic fields, while the viscoelastic shear modulus $G_{\mathrm{v}}$ and the field-induced shear modulus $G_{\mathrm{m}}$ increase with the increasing magnetic fields; it can be deduced that the increase of model parameters $G_{\mathrm{v}}$ and $G_{\mathrm{m}}$ will generally slow down until the magnetic saturation. 


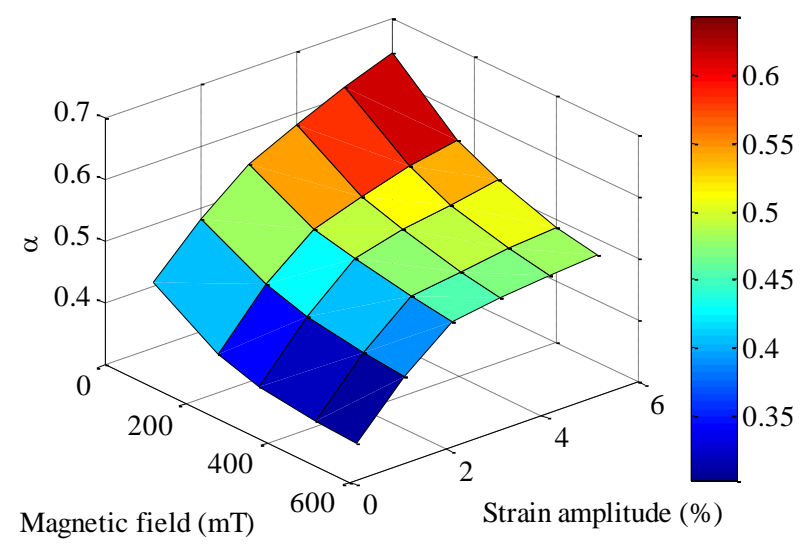

(a)

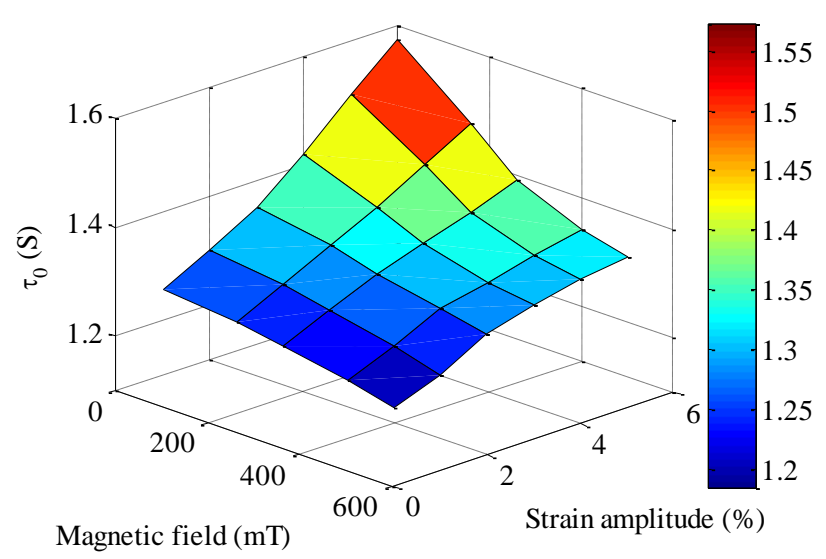

(b)

Figure 6 The magnetic field and strain amplitude dependence of parameters (a) $\alpha$ and (b) $\tau_{0}$.

Figure 8 displays an excellent agreement between the fitted results and the experimental results on the magnetic field dependence of dynamic moduli with varying strain amplitude. The storage modulus of MREs increases with the magnetic flux density, and this increase can be deduced to continue until the magnetic saturation. Both the storage modulus and loss modulus decrease with strain amplitudes, and the storage modulus increases with the frequency. The loss modulus increases with the magnetic flux density initially, reaches a maximum value around $300 \mathrm{mT}$, and decreases with the magnetic flux density afterwards. 


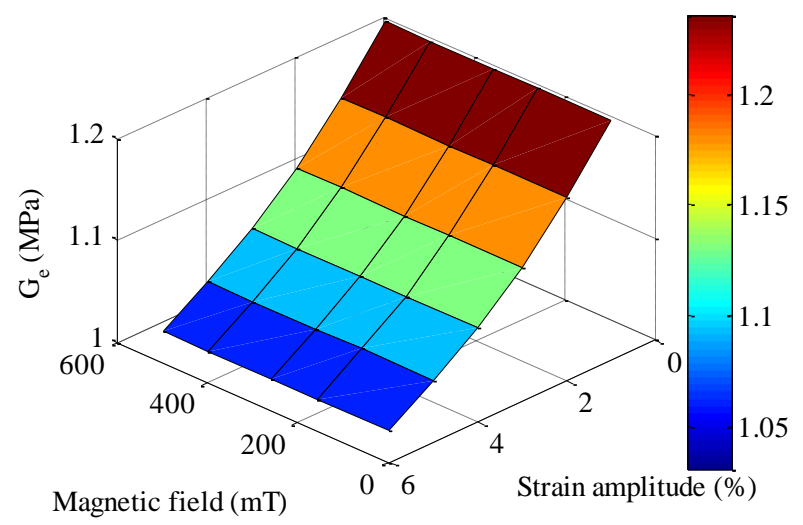

(a)

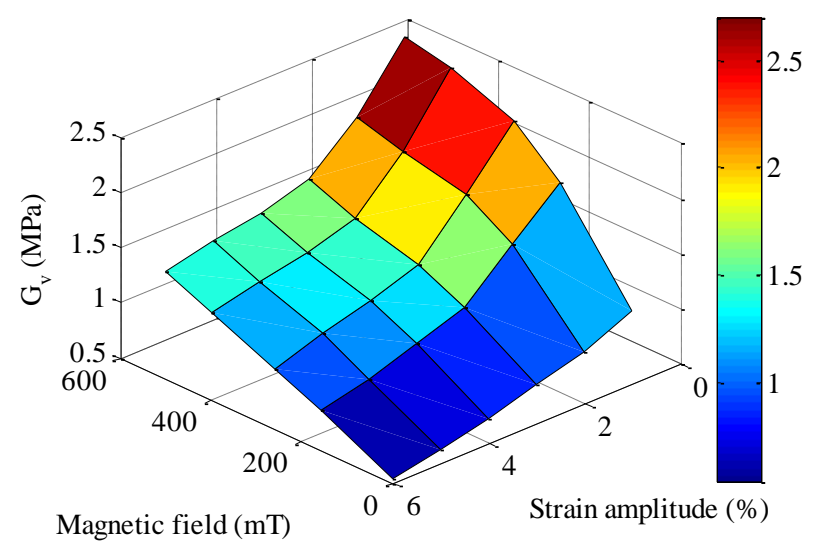

(b)

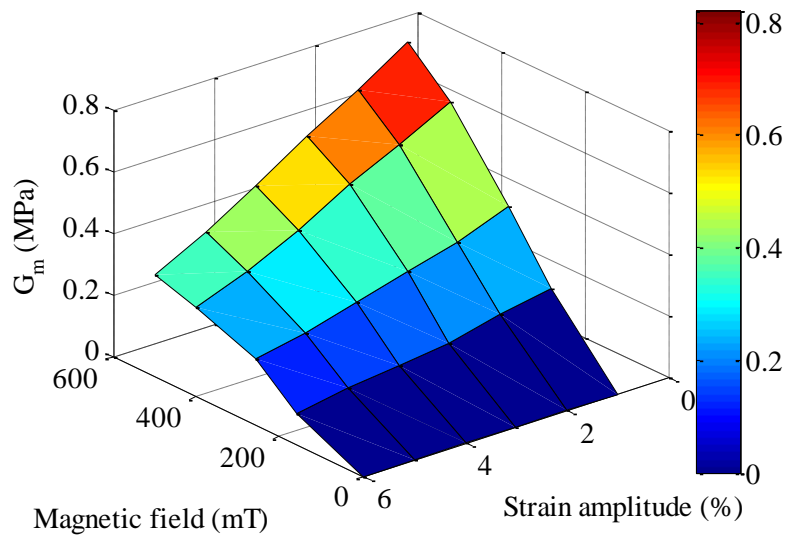

(c)

Figure 7 The magnetic field and strain amplitude dependence of parameters (a) $G_{\mathrm{e}}$, (b) $G_{\mathrm{v}}$ and (c) $G_{\mathrm{m}}$. 


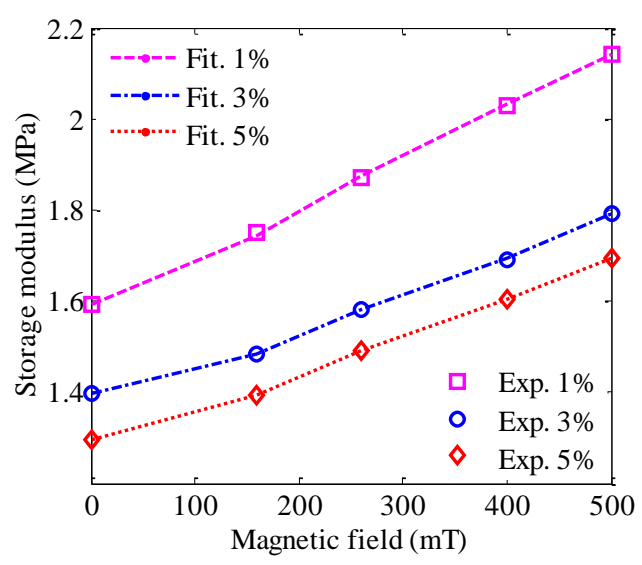

(a)

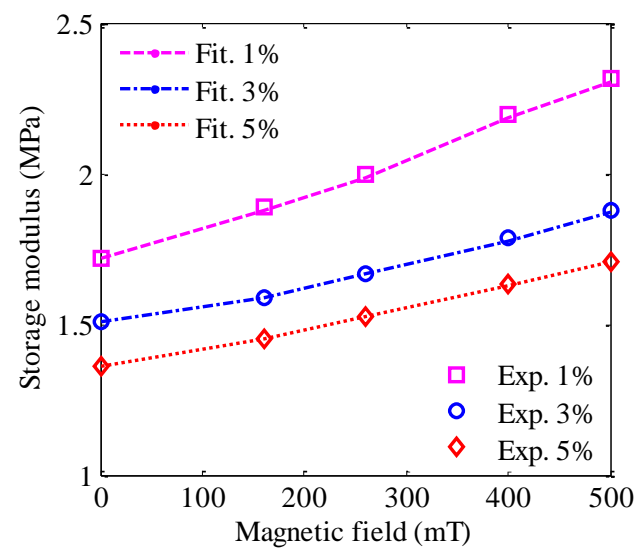

(c)

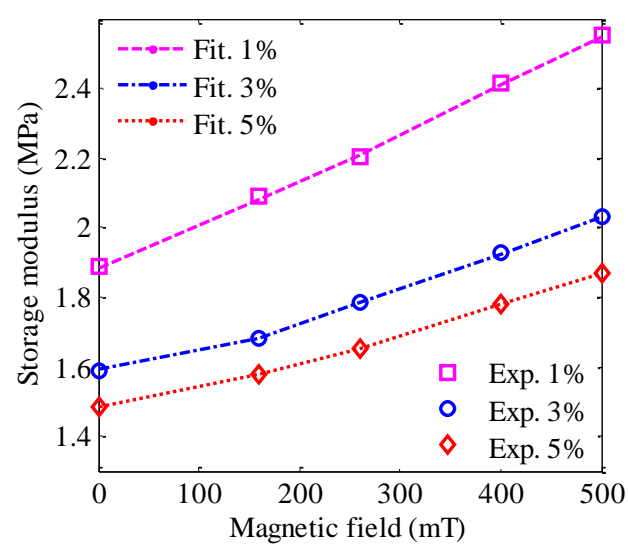

(e)

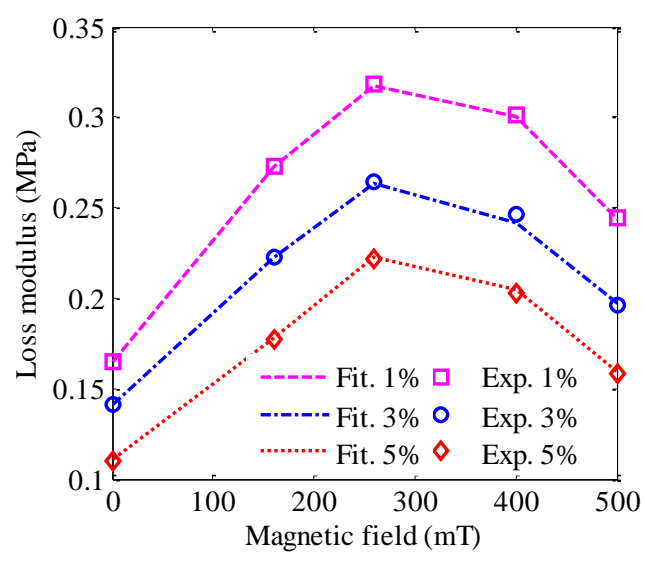

(b)

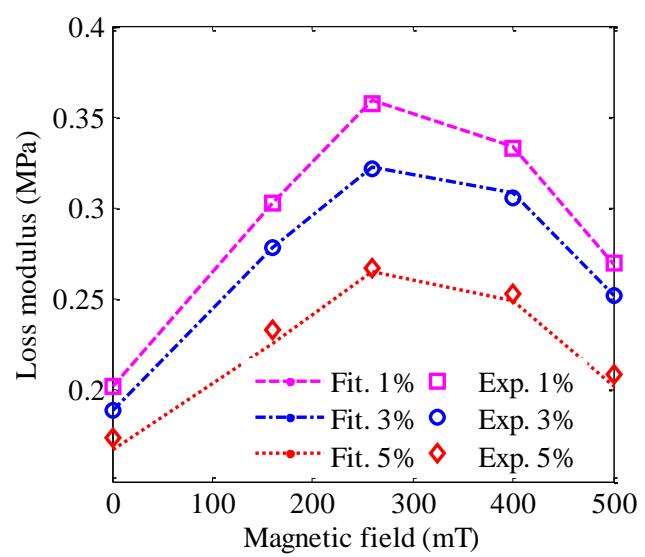

(d)

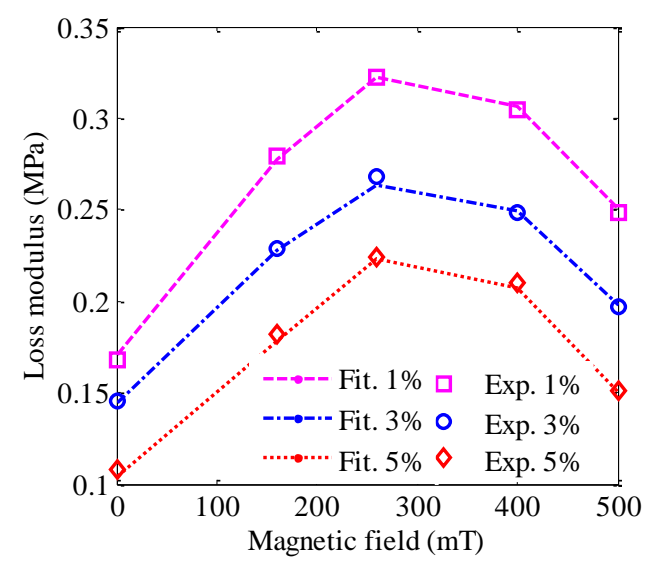

(f)

Figure 8 Comparison between experimental results and fitted results on the magnetic field dependence of storage modulus at (a) $1 \mathrm{~Hz}$, (c) $10 \mathrm{~Hz}$, (e) $50 \mathrm{~Hz}$ and loss modulus at (b) $1 \mathrm{~Hz}$, (d) $10 \mathrm{~Hz}$, (f) $50 \mathrm{~Hz}$.

\section{Numerical solution and validation}

Comparing with Riemann-Liouville and Grünwald-Letnikov fractional derivatives, the initial conditions of Caputo fractional derivative cases can own better-understood physical meanings. Because the initial conditions, such as function 
values themselves and integer-order derivatives can be mostly measured in practice, the Caputo derivative is commonly chosen to deal with viscoelastic materials. Utilizing the predictor-corrector approach to numerically solve Caputo fractional differential equations (Diethelm et al., 2002; Li and Deng, 2007), the function $f$ is assumed to exist in an interval $[0, T]$ as a unique solution, and $T$ can be taken as the integral interval. This technique is running in a uniform grid $\left\{t_{\mathrm{n}}=n h: n=0,1, \ldots, N\right\}$ with a certain integer $N$ and $h=T / N$. The predicted value and the corrector formula can be calculated as:

$$
\begin{aligned}
& x_{h}^{p}\left(t_{n+1}\right)=x_{k=0}^{[11} x_{0}^{(k)} \frac{t_{n+1}^{k}+\frac{h}{k !}}{(\quad)} a_{j=0}^{n} a_{j, n+1} f\left(t_{j}, x_{h}\left(t_{j}\right)\right) \\
& x_{h}^{c}\left(t_{n+1}\right)={ }_{k=0}^{[11} x_{0}^{(k)} \frac{t_{n+1}^{k}}{k !}+\frac{h}{(+2)} f\left(t_{n+1}, x_{h}^{P}\left(t_{n+1}\right)\right)+\frac{h}{(+2)}_{j=0}^{n}{ }_{j, n+1} f\left(t_{j}, x_{h}\left(t_{j}\right)\right)
\end{aligned}
$$

where

$$
\begin{aligned}
& \left.\left.a_{j, n+1}=\frac{h}{((n} \quad j+1\right) \quad\left(\begin{array}{ll}
n & j
\end{array}\right)\right), 1 \quad j \quad n \\
& j, n+1=\left\{\begin{array}{l}
n^{+1} \quad\left(\begin{array}{ll}
n & )(n+1), j=0 \\
\left(\begin{array}{ll}
n & j+2
\end{array}\right)^{+1}+\left(\begin{array}{ll}
n & j
\end{array}\right)^{+1} & 2\left(\begin{array}{ll}
n & j+1
\end{array}\right)^{+1}, 1 \leq j \leq n \\
1, j=n+1
\end{array}\right.
\end{array}\right.
\end{aligned}
$$

Then Equation (7) can be readily rewritten as fractional differential equations. Using the identified parameters in Figure 6, the numerical solutions can be calculated as predicted results of this nonlinear dynamic model.

$$
\begin{aligned}
& (t)=G_{e}(t)+G_{m}(t)+G_{v} \quad D_{v}(t) \\
& D_{v}(t)=\left[\begin{array}{ll}
(t) & { }_{v}(t)
\end{array}\right] / /_{0}
\end{aligned}
$$

Figure 9 demonstrates the experimental results, and the predicted results of this nonlinear dynamic model and the revised Bouc-Wen model (referred to Appendix A). The stress response of MRE samples lags behind the strain input with a loss angle when the excitation is sinusoidal, as a result the relationship between stress and strain seems approximately elliptical in shape. The comparison of stress-strain hysteresis loops under varying magnetic field, frequency and strain amplitude indicates that the experimental results support the predicted results of the proposed dynamic model very well. Furthermore, this fractional-order nonlinear model can have predicted results closer to experimental results rather than the revised Bouc-Wen model, and will be 
expected to perform better from the prospect of efficiency.

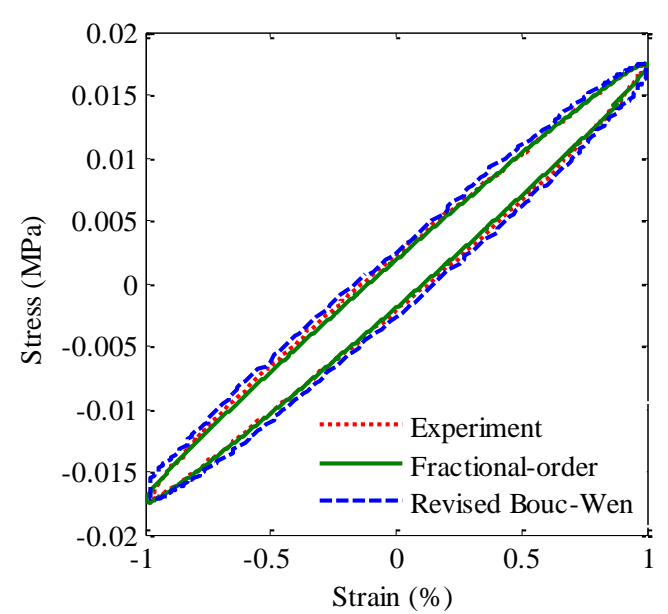

(a)

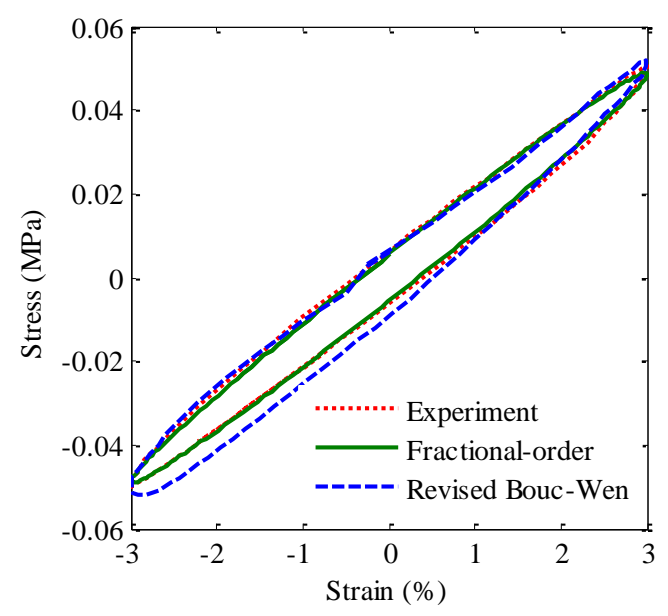

(c)

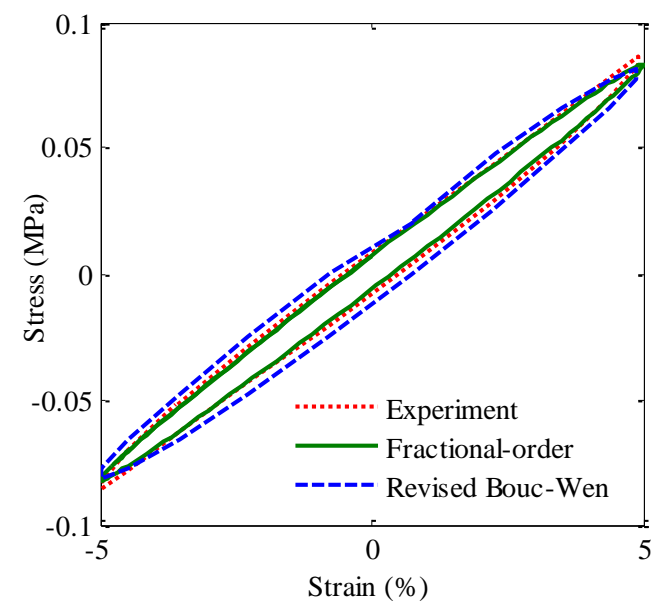

(e)

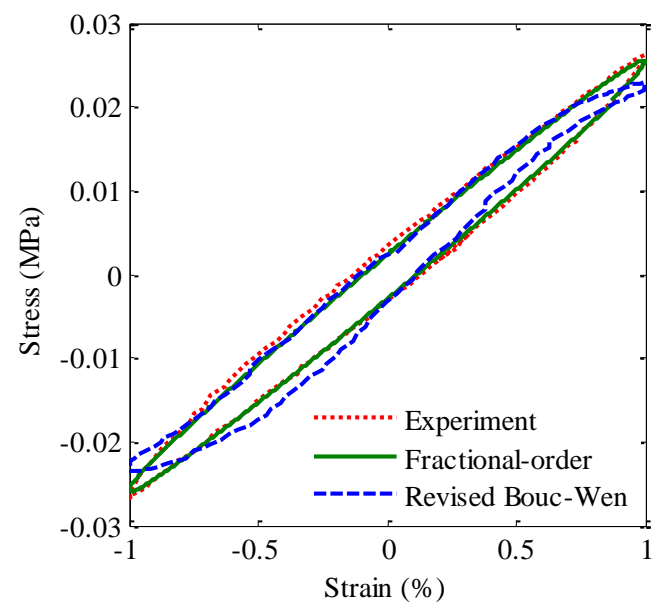

(b)

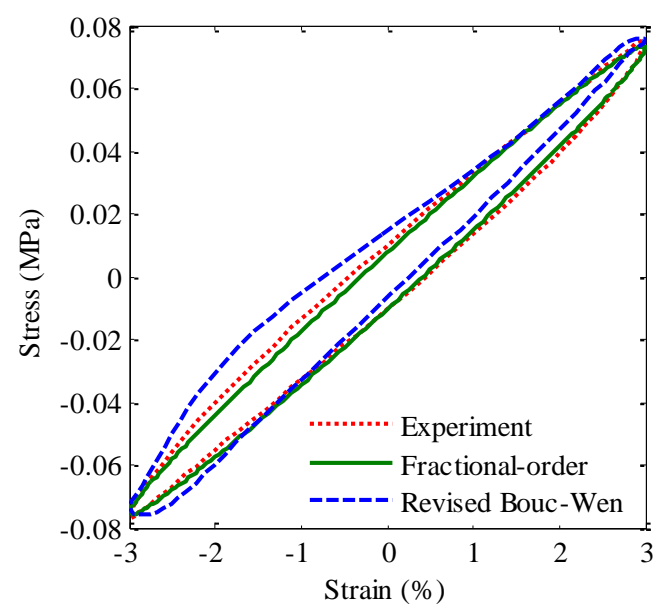

(d)

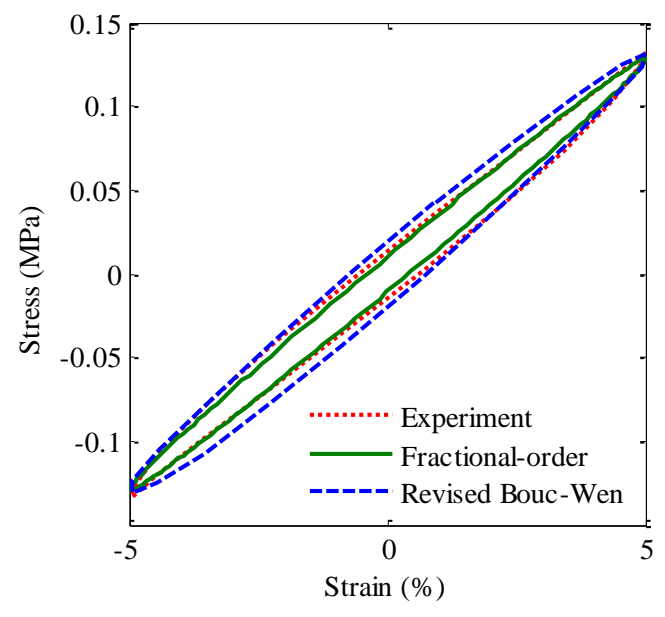

(f)

Figure 9 Comparison of stress-strain hysteresis loops between the proposed fractional-order nonlinear model and the revised Bouc-Wen model at $1 \mathrm{~Hz}$, a strain amplitude of $1 \%$ and a magnetic field of (a) 0 and (b) $500 \mathrm{mT}$, at $10 \mathrm{~Hz}$, a strain amplitude of $3 \%$ and a magnetic field of (c) 0 and (d) $500 \mathrm{mT}$, and at $50 \mathrm{~Hz}$, a strain amplitude of $5 \%$ and a magnetic field of (e) 0 and (f) $500 \mathrm{mT}$. 
The mean squared error (MSE) $S_{\mathrm{e}}{ }^{2}$ can be compared for the two models as presented in Figure 10, where this fractional-order nonlinear model is also expected to own a much higher precision accuracy. Considering the experimental data was obtained with small amplitudes to keep MRE samples glued onto clamps during DMA tests, it is evident that this proposed model possesses a high efficiency with amplitudes below $6 \%$. The energy enclosed by a stress-strain hysteresis loop is also small in MRE materials, so this proposed model is convincingly effective for materials with more elasticity than viscosity. The application of this fractional-order nonlinear model can be extended appropriately, such as to excitations of large amplitudes or other hysteresis phenomenon, but the experimental validation is essential for the expectation.

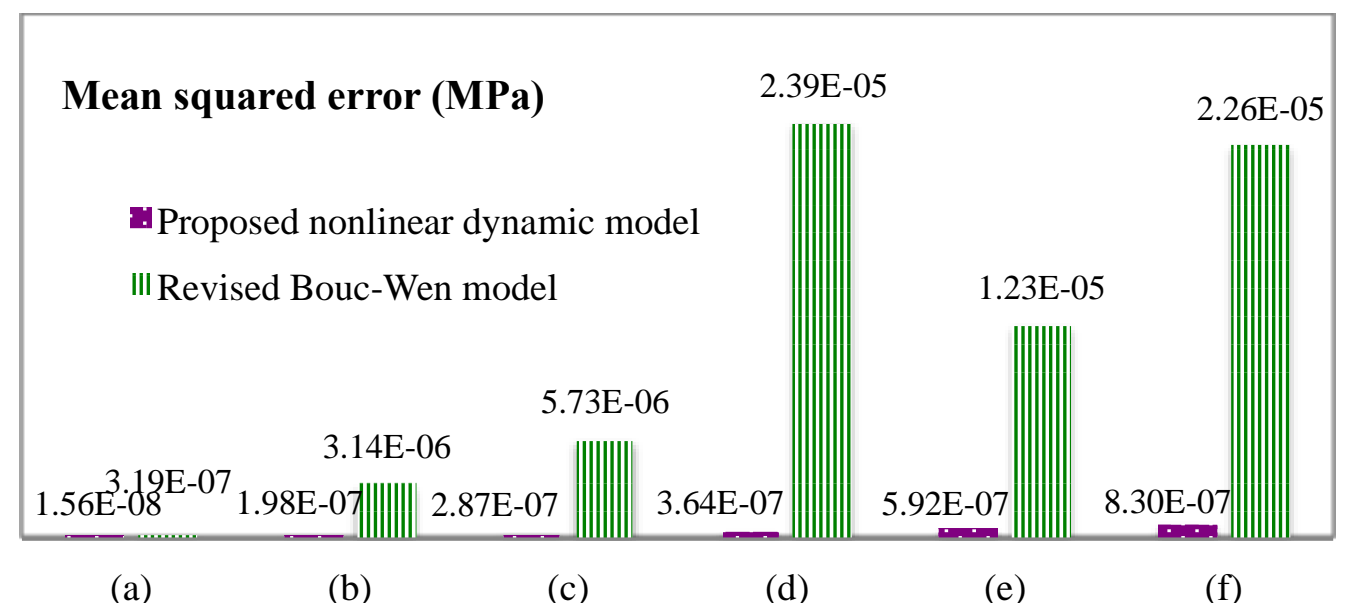

Figure 10 The comparison of $S_{\mathrm{e}}{ }^{2}$ of this proposed nonlinear dynamic model and the revised Bouc-Wen model at $1 \mathrm{~Hz}$, a strain amplitude of $1 \%$ and a magnetic field of (a) 0 and (b) $500 \mathrm{mT}$, at $10 \mathrm{~Hz}$, a strain amplitude of $3 \%$ and a magnetic field of (c) 0 and (d) $500 \mathrm{mT}$, and at $50 \mathrm{~Hz}$, a strain amplitude of $5 \%$ and a magnetic field of (e) 0 and (f) $500 \mathrm{mT}$.

\section{Conclusions}

In this study, a nonlinear model was proposed for MREs to represent the dynamic behavior by incorporating the field-induced modulus into a fractional viscoelastic model. The frequency, amplitude and magnetic field dependence of dynamic mechanical properties was experimentally studied in shear mode; and the proposed model requires fewer parameters that were identified by fitting the experimental data on dynamic moduli in frequency domain. The superior rationality of this fractional-order nonlinear model was supported with very good consistency and repeatability of the identified parameters. The magnetic field and strain amplitude dependence of model parameters was further analyzed to gain insight into the material elasticity and viscosity of MREs, and the fitted results were in agreement with the 
experimental results on the strain amplitude and magnetic field dependence of dynamic moduli. Numerical results of this proposed nonlinear dynamic model were calculated using the predictor-corrector approach, and the predicted results on the stress-strain hysteresis loop exhibited an excellent agreement with the experimental results. This nonlinear dynamic model proved to own a higher efficiency by comparing with the revised Bouc-Wen model, and an advantageous applicability for designing MRE based structures and devices.

\section{Acknowledgement}

This research was supported by National Natural Science Foundation of China (grant numbers 11702213, 11802229 and 11972282) and Science Foundation of Education Department of Shaanxi (grant number 20JK0777).

\section{References}

Bai XX, Cai FL and Chen P (2019) Resistor-capacitor (RC) operator-based hysteresis model for magnetorheological (MR) dampers. Mechanical Systems and Signal Processing 117(2): 157-169.

Behrooz M, Wang XJ and Gordaninejad F (2014) Modeling of a new semi-active/passive magnetorheological elastomer isolator. Smart Materials and Structure 23(4): 045013.

Blom P and Kari L (2011) A nonlinear constitutive audio frequency magneto-sensitive rubber model including amplitude, frequency and magnetic field dependence. Journal of Sound and Vibration 330(5): 947-954.

Chen L, Gong XL and Li WH (2007) Microstructures and viscoelastic properties of anisotropic magnetorheological elastomers. Smart Materials and Structure 16(6): 2645-2649.

Daley S and Wang J (2008) A geometric approach to the design of remotely located vibration control systems. Journal of Sound and Vibration 318(4-5): 702-714.

Dargahi A, Rakheja S and Sedaghati R (2019) Development of a field dependent Prandtl-Ishlinskii model for magnetorheological elastomers. Materials and Design 166(3): 107608.

Davis LC (1999) Model of Magnetorheological Elastomers. Journal of Applied Physics 85(6): 3348-3351.

Diethelm K, Ford NJ and Freed AD (2002) A Predictor-Corrector Approach for the Numerical Solution of Fractional Differential Equations. Nonlinear Dynamics 29(4): 3-22.

Eem SH, Koo JH and Jung HJ (2019) Feasibility study of an adaptive mount system based on magnetorheological elastomer using real-time hybrid simulation. Journal of Intelligent Material Systems and Structures 30(5): 701-707.

Fu J, Dai Z, Yang Z, et al. (2020) Time delay analysis and constant time-delay compensation control for MRE vibration control system with multiple-frequency excitation. Smart Materials and Structures 29(1): 014001.

Graczykowski C and PawlOwski P (2017) Exact physical model of magnetorheological damper. Applied Mathematical Modelling 47(7): 400-424. 
Ibrahim RA (2008) Resent advances in nonlinear passive vibration isolators. Journal of Sound and Vibration 314(3-5): 371-452.

Jolly MR, Carlson JD and Munoz BC (1996) A model of the behavior of magnetorheological materials. Smart Materials and Structures 5(5): 607-614.

Kaleta J, Królewicz M and Lewandowski D (2011) Magnetomechanical properties of anisotropic and isotropic magnetorheological composites with thermoplastic elastomer matrices. Smart Materials and Structures 20(8): 085006.

Kumbhar SB, Chavan SP and Gawade SS (2018) Adaptive tuned vibration absorber based on magnetorheological elastomer-shape memory alloy composite. Mechanical Systems and Signal Processing 100(2): 208-223.

Lerner AA and Cunefare KA (2007) Performance of MRE-based vibration absorbers. Journal of Intelligent Material Systems and Structures 19(5): 551-563.

Leng D, Xiao H, Sun L, et al. (2019) Study on a magnetorheological elastomer-base device for offshore platform vibration control. Journal of Intelligent Material Systems and Structures 30(2): 243-255.

Li C and Deng W (2007) Remarks on fractional derivatives. Applied Mathematics and Computation 187(2): 777-784.

Nguyen XB, Komatsuzaki T, Iwata Y, et al. (2018) Modeling and semi-active fuzzy control of magnetorheological elastomer-based isolator for seismic response reduction. Mechanical Systems and Signal Processing 101(2): 449-466.

Nadzharyan TA, Kostrov SA, Stepanov GV, et al. (2018) Fractional rheological models of dynamic mechanical behavior of magnetoactive elastomers in magnetic fields. Polymer 142(4): 316-329.

Nguyen XB, Komatsuzaki T and Zhang N (2020) A nonlinear magnetorheological elastomer model based on fractional viscoelasticity, magnetic dipole interactions, and adaptive smooth Coulomb friction. Mechanical Systems and Signal Processing 141(6): 106438.

Poojary UR and Gangadharan KV (2018) Integer and fractional order-based viscoelastic constitutive modeling to predict the frequency and magnetic field-induced properties of magnetorheological elastomer. Journal of Vibration and Acoustics 140(4): 041007.

Sapouna K, Xiong YP and Shenoi RA (2017) Dynamic mechanical properties of isotropic/anisotropic silicon magnetorheological elastomer composites. Smart Materials and Structures 26(10): 115010.

Sasso M, Palmieri G and Amodio D (2011) Application of fractional derivative models in linear viscoelastic problems. Mechanics of Time-Dependent Materials 15(4): 367-387

Sun SS, Yang J, Li WH, et al. (2017) Development of an isolator working with magnetorheological elastomers and fluids. Mechanical Systems and Signal Processing 83(1): 371-384.

Wan YX, Xiong YP and Zhang SM (2019) Temperature effect on viscoelastic properties of anisotropic magnetorheological elastomers under compression. Smart Materials and Structures 28(1): 015005.

Wang B and Kari L (2019) A nonlinear constitutive model by spring, fractional derivative and modified bounding surface model to represent the amplitude, frequency and the magnetic dependency for Magneto-sensitive rubber. Journal of Sound and Vibration 438(1): 344-352. 
Wang Q, Dong XF, Li LY, et al. (2018) Mechanical modeling for magnetorheological elastomer isolators based on constitutive equations and electromagnetic analysis, Smart Materials and Structures 27(6): 065017.

Xin FL, Bai XX and Qian LJ (2017) Principle, modeling and control of a magnetorheological elastomer dynamic vibration absorber for powertrain mount systems of automobiles. Journal of Intelligent Material Systems and Structures 28(16): 2239-2254.

Xing ZW, Yu M, Fu J (2015) A laminated magnetorheological elastomer bearing prototype for seismic mitigation of bridge superstructures. Journal of Intelligent Material Systems and Structures 26(14): 1818-1825.

Yang J, Du H, Li W, et al. (2013) Experimental study and modeling of a novel magnetorheological elastomer isolator. Smart Materials and Structures 22(11): 117001.

Yu Y, Li Y and Li J (2015) Parameter identification of a novel strain stiffening model for magnetorheological elastomer base isolator utilizing enhanced particle swarm optimization. Journal of Intelligent Material Systems and Structures 26(18): 2446-2462.

Yuan L, Sun SS, Pan ZG, et al. (2019) Mode coupling chatter suppression for robotic machining using semi-active magnetorheological elastomers absorber. Mechanical Systems and Signal Processing 117(2): 221-237.

Yusoff NIM, Chailleux E and Airey GD (2011) A Comparative Study of the Influence of Shift Factor Equations on Master Curve Construction. International Journal of Pavement Research and Technology 4(6): 324-336.

Zhu G, Xiong Y, Li Z, et al. (2020) A fractional-order model on the dynamic mechanical behavior of magnetorheological elastomers. Smart Materials and Structures 29(2): 025020 .

\section{Appendix}

The revised Bouc-Wen model proposed by Wang et al. (2018) is employed in this piece of work, and the constitutive equations of the MREs can be expressed as:

$$
\begin{aligned}
& \tau=k_{0} \gamma+Z+c_{0} \dot{\gamma}+m_{0} \ddot{\gamma} \\
& \dot{z}=A \dot{\gamma}-\beta|\dot{\gamma}||z|^{n-1} Z-\gamma_{\text {bw }} \dot{\gamma}|z|^{n}
\end{aligned}
$$

where $\gamma$ and $\tau$ are the shear strain and shear stress, respectively; $c_{0}$ and $k_{0}$ are the parameters of the Kelvin-Voigt model; $A, \beta, \gamma_{\mathrm{bw}}$ and $n$ are the non-dimensional parameters for regulating the stress-strain hysteresis loop in shear mode; and $z$ is the evolutionary variable. All the parameter identification is based on the experimental data by using GA.

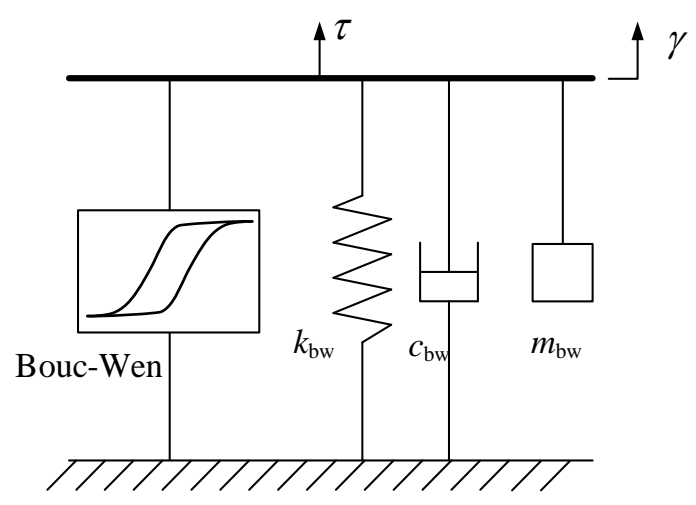

Figure A.1 Schematic of the revised Bouc-Wen model. 